\title{
Linking the Ex-Gaussian Parameters to Cognitive Stages: Insights from the Linear Ballistic Accumulator (LBA) Model
}

\author{
Daniel Fitousi ${ }^{\mathrm{a} \otimes(1)}$ \\ ${ }^{a}$ Ariel University, Israel.
}

\begin{abstract}
The ex-Gaussian distribution has been routinely used by researchers as a descriptive tool to characterize response time data in speeded two-choice tasks. It has also served as a psychological theory of the underlying cognitive processes. The latter practice requires that the exGaussian parameters should map in a one-to-one correspondence to distinct cognitive stages or process model parameters. The current investigation assessed the correspondence between the exGaussian parameters and the parameters of a well-established process model - the linear ballistic accumulator (LBA, Brown \& Heathcote, 2008), using simulations and analyses of empirical data. The results showed the ex-Gaussian parameters bear lawful relations with the LBA's parameters, but that these relations are not unique. These results are in accordance with two earlier studies by Matzke and Wagenmakers (2009) and Rieger and Miller (2019), who assessed the relations between the ex-Gaussian and the diffusion's model parameters. The outcome of the current study suggests that researchers should exercise caution when deploying the ex-Gaussian parameters as a means of psychological theorizing.
\end{abstract}

Keywords $₫$ Ex-Gaussian distribution, Linear Ballistic accumulator.

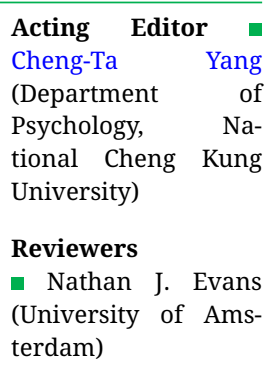

and one anonymous reviewer

\section{danielfi@ariel.ac.il}

10.20982/tqmp.16.2.p091

\section{Introduction}

Analysis of response times (RT) is a central methodology in psychology (Luce, 1986; Townsend \& Ashby, 1983) in which the mean or median RTs are routinely used as dependent variables. However, the means can conceal important patterns that lurk in the RT distributions. The last decades have seen a growing number of researchers who go beyond the mean and use the statistical properties of the RT distribution (Ratcliff, 1978; for review, see Algom, Eidels, Hawkins, Jefferson, \& Townsend, 2015). Several probability distributions have been used in cognitive science to fit RT distributions. Among these distributions are the Weibull (Logan, 1988), the shifted Wald (Schwarz, 2001), and the log-normal (Van der Linden, 2006). But the exGaussian distribution stands out as one of the most popular and influential approaches to modelling RT distributions (Ratcliff \& Murdock, 1976; Hohle, 1965; Luce, 1986). The ex-Gaussian has been used as either a descriptive tool of the RT distribution or a theoretical account of the un- derlying cognitive processes (Balota \& Spieler, 1999). In its two roles, the ex-Gaussian has been applied successfully to many research questions (Balota \& Yap, 2011), having major impact on theorizing in many areas of cognition (LethSteensen, Elbaz, \& Douglas, 2000; Possamaï, 1991; Rotello \& Zeng, 2008; Steinhauser \& Hübner, 2009; Schmiedek, Oberauer, Wilhelm, Süß, \& Wittmann, 2007; Tse, Balota, Yap, Duchek, \& McCabe, 2010).

In order to use the ex-Gaussian distribution as a theoretical account, one needs to assume that the exGaussian parameters map uniquely to cognitive stages or processes (Balota \& Yap, 2011; Ratcliff \& Murdock, 1976). This assumption has been corroborated in earlier studies (Schmiedek et al., 2007; Schwarz, 2001). However, recent studies by Matzke and Wagenmakers (2009) and Rieger and Miller (2019) have challenged this assumption. These authors have argued that the ex-Gaussian parameters do not correspond uniquely (i.e. in a one-to-one fashion) to experimental manipulations that are known to influence distinct cognitive stages. According to these researchers, 
the ex-Gaussian parameters also do not map uniquely to the parameters of a well-established process model - Ratcliff's diffusion model (Ratcliff, 1978) - and to its related offspring - the EZ diffusion (Wagenmakers, Van Der Maas, \& Grasman, 2007). These studies are important, but it is not clear whether they generalize to other process models than the diffusion model. One model that comes to mind is the linear ballistic accumulation (LBA, Brown \& Heathcote, 2008) - a prominent competitor of the diffusion model.

Albeit their similarity, the diffusion and LBA models can, under certain circumstances, lead to divergent conclusions (van Ravenzwaaij \& Oberauer, 2009; Osth, Bora, Dennis, \& Heathcote, 2017). Therefore, the goal of the current study has been to link the ex-Gaussian parameters to those of the LBA. The rationale for the current study deploys the same logic guiding Matzke and Wagenmakers (2009) and Rieger and Miller (2019) in their studies. The present study adopted the same tools deployed by these earlier studies, namely, simulations and analyses of empirical data. The work provides further evidence concerning the suitability of the ex-Gaussian parameters as a theoretical account, and sheds light on the relations between the diffusion and LBA models.

\section{The ex-Gaussian distribution}

The ex-Gaussian (see Figure 1) is a convolution of a Gaussian and an exponential distribution (Ratcliff \& Murdock, 1976; Heathcote, Popiel, \& Mewhort, 1991; Hohle, 1965; Luce, 1986). The Gaussian part is represented by two parameters, the mean $\mu$ and standard deviation $\sigma$, and the exponential part is represented by its mean $\tau$. The probability distribution function (pdf) of the function is expressed as:

$$
f(t \mid \mu, \sigma, \tau)=\frac{1}{\tau \sqrt{2 \pi}} \exp \left(\frac{\sigma^{2}}{2 \tau^{2}}-(t-\mu) / \tau\right) \times \int_{-\infty}^{(t-\mu) / \sigma-(\sigma / \tau)} \exp \left(-y^{2} / 2\right) \mathrm{d} y
$$

The mean and the variance of the ex-Gaussian function are:

$$
\begin{gathered}
E(x)=\mu+\tau \\
\operatorname{Var}(x)=\sigma^{2}+\tau^{2}
\end{gathered}
$$

The ex-Gaussian often provides excellent fit to RT distributions (Luce, 1986; Ratcliff \& Murdock, 1976). The $\mu$ and $\sigma$ reflect the location and scale of the ex-Gaussian, while the ratio $\tau / \sigma$ reflects its shape (Rouder, Lu, Speckman, Sun, \& Jiang, 2005). The quantity $2 \tau^{3}$ is its skew. Moreover, the ex-Gaussian offers a tight constrain on the mean of an empirical distribution because it is the sum of $\mu$ and $\tau$. This fact allows a direct connection to the mean RT literature.

Researchers fit the ex-Gaussian parameters to empirical distributions to assess the influence of experimental manipulation on hypothetical cognitive stages (Heathcote et al., 1991; Hohle, 1965; Luce, 1986). The major tactic is to test the influence of different experimental conditions (e.g., congruent vs. incongruent) or groups (e.g., ADHD vs. normal) on the three parameters of the ex-Gaussian distribution. In routine applications, the three ex-Gaussian parameters $\mu, \tau$, and $\sigma$ are estimated for each participant in each condition, and are then subjected to analysis (e.g.,
ANOVA) ${ }^{1}$. This approach has been applied successfully to various questions in diverse areas such as word reading (Balota, Yap, Cortese, \& Watson, 2008), individual differences in the Stroop task (Heathcote et al., 1991), memory (Hockley, 1984; Rohrer, 1996; Rohrer \& Wixted, 1994), research on ADHD (Buzy, Medoff, \& Schweitzer, 2009; LethSteensen et al., 2000) and Alzheimer's disease (Jackson, Balota, Duchek, \& Head, 2012; Gordon \& Carson, 1990).

\section{The Ex-Gaussian as a Descriptive and Theoretical Model}

As noted at the outset, the ex-Gaussian distribution can serve as a either a descriptive or a as theoretical model. Descriptive models of RT distributions, such as the Weibull (Logan, 1988) and the ex-Gaussian afford researchers the least-committed level of interpretation. They allow robust measures of location, scale, and shape (Rouder et al., 2005), and accurate measurement of the effects of manipulations and type of group, without the need to surmise specific cognitive construct or process. These descriptive measures can be used to constrain theories and motivate new ones (see Heathcote et al., 1991).

In its role as a theoretical model, the ex-Gaussian dis-

\footnotetext{
${ }^{1}$ A Reviewer rightly noted that this popular practice is problematic when insufficient amount of data is collected. Firstly, fitting distributions require thousands of trials per participant. Secondly, researchers ignore the hierarchical structure lurking in the data (i.e., trials are nested within participants), which in turn can lead to biased conclusions. Hierarchical models of the multilevel data can provide a better unbiased alternative (see Boehm, Marsman, Matzke, \& Wagenmakers, 2018; Rouder et al., 2005).
} 
Figure 1 1 The shape of the ex-Gaussian distribution as a function of changes in the ex-Gaussian parameters. $(A) \mu=400$, $\sigma=100, \tau=100$ (default parameters), (B) $\mu=800, \sigma=100, \tau=100$ (increasing $\mu$ ), (C) $\mu=400, \sigma=150, \tau=100$ (increasing $\sigma$ ), and (D) $\mu=400, \sigma=100, \tau=200$ (increasing $\tau$ ).

\section{A Default Paramter}
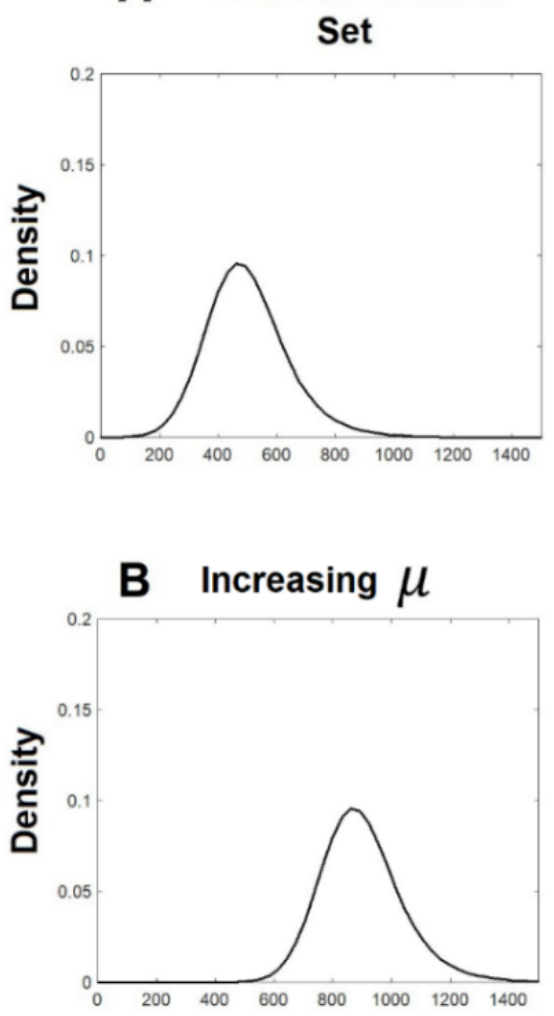

tribution has been decomposed into its Gaussian (mu and sigma) and exponential (tau) parameters, with each member of the duo being attributed to a specific type of cognitive process or construct. The Gaussian parameters mu $(\mu)$ and sigma $(\sigma)$ have been related to "more stimulus driven automatic (nonanalytic) processes whereas the $\tau$ component is related to a more central attention demanding (analytic) processes” (p.348) (Balota \& Spieler, 1999). A closely related partition has ascribed the Gaussian parameters to sensory stages, while the exponential parameter to decisional stages (Gordon \& Carson, 1990; Possamaï, 1991; Rotello \& Zeng, 2008). This may be because the exponential parameter exclusively affects the tail of the distribution. The ex-Gaussian distribution has been applied to a wide range of research questions. For example, in the domain of memory and control, it has been argued (Schmiedek et al., 2007; Tse et al., 2010) that the exponential component, tau is exclusively related to working memory capacity and control. Schmiedek et al. (2007) tested participants
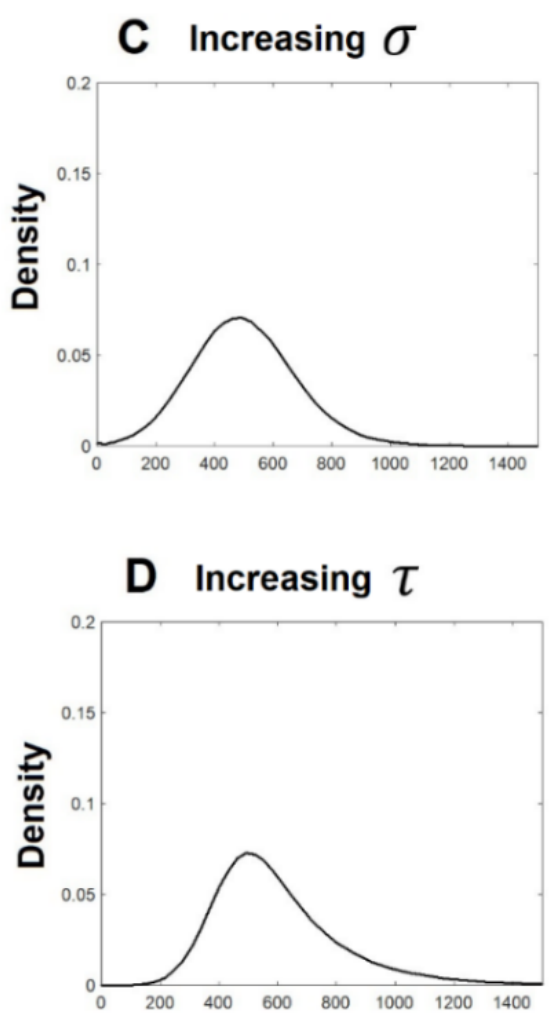

on several choice RT task which measure working memory and fluid intelligence. They revealed that individual differences in tau were correlated with working memory capacities. Similarly, Tse et al. (2010) compared performance of early dementia (DAT) patients with that of healthy patients. They found that performance of the DAT patients was predicted by their tau scores, which in return, were also correlated with psychometric measures of working memory, long-term episodic and semantic memory, and processing speed.

Another example concerns the case for tau as a potential marker of task conflict in the Stroop task (Steinhauser \& Hübner, 2009). Stroop stimuli, both congruent (RED in red) and incongruent (RED in green) call for two opposing demands - reading (the word) and naming (the ink color), while neutral stimuli (e.g. ${ }^{* * * * * * *}$ in red) do not create task conflict because they activate only the color naming task. It is often found that RTs are faster in the neutral than in the congruent condition. This effect has been ascribed to 
task conflict. While the regular Stroop effect (RT incongruent - RT congruent) manifests as an influence on the mu component, the task conflict effect is manifested only as an influence on tau component (Heathcote et al., 1991; Steinhauser \& Hübner, 2009).

For yet another example in the area of attention, consider the study by Kóbor et al. (2015). They tested children with and without ADHD in a Stroop task. At the level of the mean RTs, a Stroop effect was found for both groups, and overall ADHD children responded slower than normal children. Analyses on the ex-Gaussian parameters revealed that the Stroop effect influenced only the $\mu$ parameter, whereas the group effect was manifested only in the $\tau$, with increasing values of $\tau$ for the ADHD group. This increase has been attributed to lapses of attention in the that group (see also Leth-Steensen et al., 2000).

But can one ascribe clear-cut psychological stages/operations to the ex-Gaussian parameters? Several researchers have criticized the deployment of the ex-Gaussian distribution as a psychological model. This criticism has been largely based on the relations of the ex-Gaussian parameters to those of the diffusion model (Matzke \& Wagenmakers, 2009; Rieger \& Miller, 2019). Since the diffusion model (Ratcliff, 1978) plays a central role in the attack, and since the current study sought to extend the investigation with respect to a rival model - the LBA (Brown \& Heathcote, 2008) - the next section provides a brief introduction of the two models.

\section{The diffusion and $L B A$ models}

Sequential sampling models (s60; Usher \& McClelland, 2001) have a long tradition in psychology. They have been developed to account for the speeded performance in twochoice tasks (e.g., a lexical decision task). In this class of models, information is gradually accumulated, and each response is associated with a decision boundary. Among the various sequential sampling models developed, the diffusion decision model (DDM Ratcliff, 1978) stands out as the most effective in accounting for two-choice behavior (for reviews see Forstmann, Ratcliff, \& Wagenmakers, 2016; Ratcliff, Smith, Brown, \& McKoon, 2016; Ratcliff \& Smith, 2004). The model assumes that evidence is continuous and varies momentarily during accumulation (i.e., stochastic). The DDM has four major parameters: drift rate $(v)$, threshold $(a)$, starting point $(z)$ and nondecision time $\left(t_{0}\right)$. The drift rate quantifies the mean rate of evidence accumulation which is affected by the quality of the stimulus or task difficulty. Threshold measures the separation between the two response boundaries and is affected by response caution. A high threshold entails that more evidence should be accumulated before the decision bound is reached. The starting point parameter quantifies the initial evidence value before accumulation starts, reflecting participant's a priori response bias. Nondecision time measures the duration of processes that include encoding time and response execution. Nondecision time varies between trials uniformly over range St. The RT equals the sum of nondecision time and the decision time which is the time it takes the accumulator to reach the boundary. The model can account for RTs in error trials and correct trials. Additional parameters include Sv, a Gaussian variability with standard deviation, according to which the drift rate varies, and Sz, a uniform range over which the starting point varies from trial-to-trial.

Brown and Heathcote (2008) have proposed a competing model to the DDM (Ratcliff, 1978), which they term the linear ballistic accumulation model (LBA, Brown \& Heathcote, 2008). There are two major features that differentiate the LBA from the diffusion model. First, the LBA assigns a separate accumulator to each possible response. So, for example, in a lexical decision task, one accumulator accrues evidence in favour of the "word" response and another accumulates evidence in favor of the "non-word" response (see Figure 2B). Second, unlike in the diffusion model, accumulation of evidence does not vary from moment-tomoment but propagates in a linear fashion (see Figure 2, bottom panel).

The activation in each accumulator starts at a value randomly sampled from the interval $[0, A]$. The accumulation of evidence is ballistic (noiseless) and linear. Each accumulator is associated with an average slope called drift rate, $v_{1}$, and $v_{2}$. These drift rates vary from trial-totrial with a standard deviation s. When evidence in either of the accumulators reaches its threshold, b1 or b2, the response is made. The model also assumes that nondecision processes take time $T_{e r}$. The LBA has been applied successfully to many research questions, accounting very well for both correct and incorrect RTs in both binary and multiple-choice tasks (Donkin, Brown, \& Heathcote, 2011; Forstmann et al., 2016).

\section{Links between the ex-Gaussian and diffusion model pa- rameters}

At first look, one may justly ask why should the parameters of an a-theoretic model such as the ex-Gaussian be related to high cognitive functions? Even researchers who are sympathetic with the idea that the ex-Gaussian can guide theoretical inferences have acknowledged that: "a theoretical interpretation of the ex-Gaussian parameters is not straightforward" (Schmiedek et al., 2007, p. 415). The first attempts to demonstrate a linkage between the ex-Gaussian distribution parameters and cognitive stages relied on the diffusion model (Schwarz, 2001).

Simulations by Schwarz (2001) have demonstrated the 
Figure 2 - Schematic illustrations of the diffusion (panel A) and LBA (panel B) models.

A Diffusion

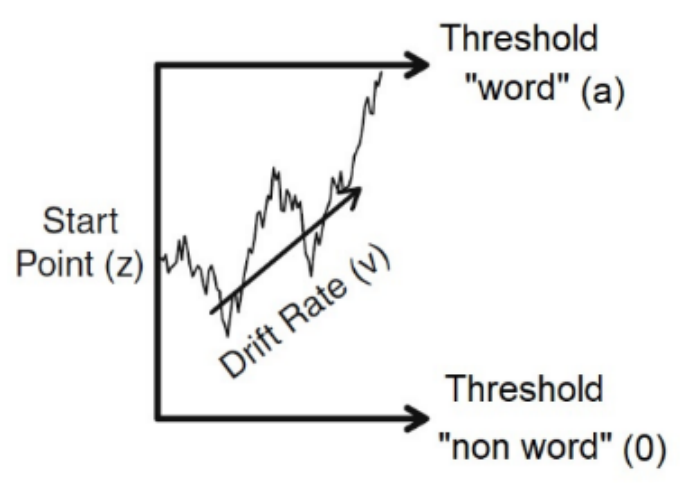

LBA

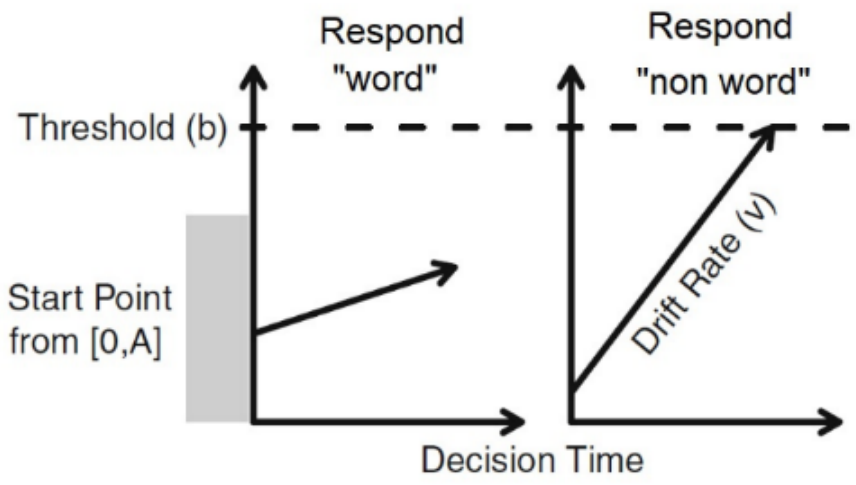

tau $\tau$ component to be strongly related to the drift rate, with larger values of drift rate leading to smaller values of $\tau$, whereas $\mu$ was found to be related to response criterion, with less conservative values of response criterion related to smaller estimates of $\mu$. In a more sophisticated approach, Schmiedek et al. (2007) investigated a network of hypotheses, linking the ideas that: (a) working memory performance is related to the slower RTs portion of the RT distribution, a hypothesis known as the worst performance rule (Coyle, 2003), (b) the hypothesis that $\tau$ should be related to working memory through (a), and (c) the hypothesis that both working memory performance and $\tau$ should be related to changes in the drift parameter of the EZ-diffusion. Using structural equation modeling (SEM), these researchers found strong evidence for this network of hypotheses.

The studies by Schmiedek et al. (2007) and Schwarz (2001) provide substantial support in the idea that the exGaussian parameters can be mapped to distinct cognitive processes, and particularly to the parameters of the EZdiffusion and diffusion models. However, two recent studies by Matzke and Wagenmakers (2009) and Rieger and Miller (2019) raised strong reservations against the practice of using the ex-Gaussian parameters as a psychological theory. These researchers have argued that the exGaussian parameters are not selectively influenced by experimental manipulations, showing that each of the exGaussian parameters map to more than one distinct processing stages. In one part of the Matzke and Wagenmakers (2009) study, they simulated RT distributions from known parameters in the DDM and then fit the ex-Gaussian parameters to them. They then plotted each of the ex-
Gaussian parameter against each of the four central DDM parameter. They did find consistent relations between the two types of parameters. However, the parameters of the ex-Gaussian did not map uniquely to those of the diffusion model (Ratcliff, 1978). Of course, for this practice to be valid one must assume that the DDM's parameters map directly to distinct cognitive stages (Voss, Rothermund, \& Voss, 2004). In a second part of their study, they fit the exGaussian parameters to published data that featured manipulations of task difficultly, response caution, and a priori bias. Prior analyses showed that each of the latter manipulations affected a unique diffusion model parameter (Voss et al., 2004). In both cases, they found the associations between the parameters were not unique. In particular, the ex-Gaussian parameter, $\mu$ was influenced by the diffusion model parameters a, starting point $\mathrm{z}$, and nondecision time $T_{e r}$. On the other hand, $\tau$ was sensitive to changes in both drift rate $\mathrm{v}$ and boundary separation $\mathrm{a}$. These results were taken as strong evidence against the practice of assigning cognitive meaning to ex-Gaussian parameters.

The study by Rieger and Miller (2019) tested a similar hypothesis regarding the linkage between model parameters and cognitive stages using the ex-Gaussian, the exWald (Schwarz, 2001), and the EZ diffusion model (Wagenmakers et al., 2007). The authors fit these models to several experiments in which hand vs. foot responses were compared. The effect on RTs in these studies could be safely attributed to the response stage. But neither of the tested models exhibit a clear linkage of its parameters to motor and pre-motor stages. When Rieger and Miller plotted the values of EZ-diffusion against the ex-Gaussian 
parameters they found $\mu$ to be correlated with the nondecision time $T_{e r}$ and $\tau$ to be correlated with the decision time Td. The decision time parameter in the EZ-diffusion model is strongly related to the drift rate parameter (see also Schmiedek et al., 2007), but it is also affected by other parameters (e.g., a boundary separation).

In sum, both studies have cast serious doubts on the psychological validity of the ex-Gaussian parameters based on their results from the diffusion model. However, an obvious fact that has been often overlooked in these studies, but should be stated from the outset, is that mathematically speaking, it is impossible to have a bijective mapping between the parameters of the two models (the diffusion and the ex-Gaussian). This is an inevitable consequence of the structural differences between the diffusion model and the ex-Gaussian distribution. The diffusion model has 7 parameters to describe two RT distributions (error and correct responses), but the ex-Gaussian, applied to each distribution separately, has only 6 parameters in total. Similarly, the LBA can be fitted to a 2AFC setting, whilst the ex-Gaussian distribution can only be fitted to a single experimental condition at a time. The best thing that can be hoped for in both cases, is that a subset of the diffusion (or in the current work, LBA) parameters would exhibit a oneto-one correspondence with the ex-Gaussian parameters.

\section{The current study}

The present study sought to test whether the ex-Gaussian parameters can be linked in a one-to-one correspondence to the parameters of the LBA (Brown \& Heathcote, 2008). The current investigation follows the exact methodological procedures performed by Matzke and Wagenmakers (2009). It also uses the same published data sets they have analyzed. In study 1, I generated RT distribution from LBA models with known parameters and then fit the exGaussian parameters to them, assessing the associations between each ex-Gaussian parameter and the LBA parameters. In study 2, I fit both LBA and ex-Gaussian models to published data by Wagenmakers, Ratcliff, Gomez, and McKoon (2008), assessing the relations between the parameters of the two models, and their relations to the original experimental manipulations they were subjected to.

\section{Study 1}

In each simulation, the pertinent LBA parameter (e.g., $v$ ) was increased in small steps while all the other four parameters $\left(A, b, s, T_{e r}\right)$ were held fixed. ${ }^{2}$ The ranges of values of the fixed and varied parameters are presented in Table 1. These ranges were used by (Donkin, Brown, Heathcote, \& Wagenmakers, 2011) in simulations. The general procedure consisted of: (a) increasing the value of the pertinent LBA parameter, (b) generating a synthetic RT distribution, and (c) fitting the ex-Gaussian parameters to this RT distribution, (d) repeating (a)- (c), each time, increasing the relevant LBA parameter in small amount and deriving the best fitting ex-Gaussian parameters. I have also made the common assumption that the non-decision parameter $T_{e r}$, the starting point $A$, and the standard deviation of the drift rates $s$ are equal for the two accumulators (see also Brown \& Heathcote, 2008; Donkin, Brown, \& Heathcote, 2011). The simulations focused on the case where the decision process is unbiased, with the assumption that the average distance from the start point to the response criterion is equal for the two accumulators (i.e., $b_{1}=b_{2}$ ). I run simulations twice. In the first version, the mean drift rates of the two LBA accumulators sum up to one $\left(v_{1}+v_{2}=1\right.$ ) (see also Donkin, Brown, \& Heathcote, 2011). In the second version, the mean drift rates did not sum up to (i.e., $v_{1}+v_{2} \neq 1$ ) because $v_{2}$ was fixed to a single unchanging value (0.2).

Along these lines, there were 4 separate simulations, one for each of the four LBA parameters ( $v, A, T_{e r}$, and $b$ [ $s$ was always held fixed]). In each simulation, the pertinent LBA parameter (e.g., $v$ ) was changed in small steps while all the other four parameters $\left(A, b, s, T_{e r}\right)$ were held fixed. After increasing the value of the pertinent LBA parameter, I generated a synthetic RT distribution with 10,000 samples from the resulting LBA model. Then, I fit the ex-Gaussian parameters ( $\mu, \sigma$, and $\tau$ ) to this RT distribution. This procedure was repeated 1000 times, each time, increasing the relevant LBA parameter in small amount and deriving the best fitting ex-Gaussian parameters $(\mu, \sigma$, and $\tau)$. I then plotted for each simulation and ex-Gaussian parameter $(\mu$, $\sigma$, and $\tau$ ), the value of the ex-Gaussian parameter against the value of the pertinent LBA parameter (see Figure 3 and 4).

The procedure of fitting ex-Gaussian distribution consisted of finding the values of the ex-Gaussian parameters: $\mu(\mathrm{mu})$, sigma $(\sigma)$, and tau $(\tau)$ that best predicted the observed (LBA generated) data. The fitting procedure was performed on RT distributions excluding incorrect or very long RTs (>4000 ms). The fitting procedure consisted of two stages. At a first stage, initial parameter values were identified using a grid-search procedure. At a second stage, these values were fed into a SIMPLEX optimization algorithm (Nelder \& Mead, 1965). The validity of the fitting method used here was confirmed in a parameter recovery study (see Supplementary Materials). It should be mentioned that more modern methods exist for optimizing the parameter values of sequential sampling models which can move through the correlated dimensions often seen in

\footnotetext{
${ }^{2}$ It should be noted that more advanced methods of covering the parameter space, such as Latin hypercube sampling do exit (Evans, Holmes, \& Trueblood, 2019).
} 
these models (Evans, 2019; Evans \& Servant, 2020).

Assessment of fit was achieved by superimposing the estimated ex-Gaussian function on a histogram of the corresponding data to the $0.1,0.3,0.5,0.7$, and 0.9 quantiles and computing the chi-square (Heathcote et al., 1991). Best-fitting parameters were those that minimized the chisquare function.

The values of best fitting ex-Gaussian parameters were plotted against their corresponding LBA parameters for the case in which the two mean drift rates sum up to 1 (Figure 3), and for the case where the two mean drift rates did not sum up to 1 (Figure 4). The results in the two cases were highly comparable. The LBA and the ex-Gaussian parameters exhibited lawful relations in most cases. But these relations were not unique because most of the LBA parameters corresponded to more than one ex-Gaussian parameter, and all ex-Gaussian parameters corresponded to more than one LBA parameter. The boundary separation (b) was strongly and positively related to $\mu$ and $\tau$ and moderately and positively to $\sigma$. The starting point ( $A$ ) was strongly and negatively related to $\mu$ and moderately and positively to $\sigma$ but had no relations with $\tau$. The drift rate parameter (v) was strongly and negatively related to $\mu$ and $\tau$. The only LBA parameter that exhibited an association with a single ex-Gaussian parameter was the non-decision time $\left(T_{e r}\right)$.

Table 2 summarizes the observed relations between the ex-Gaussian and LBA parameters along with those found for the diffusion model by Matzke and Wagenmakers (2009). Notably, the parameters of the two models show high level of agreement. Both decision bounds parameters exhibit a substantial positive association with $\mu$ and $\tau$, and a weak positive association with $\sigma$. Both non-decision time parameters exhibit substantial positive relations with $\mu$, and no relations with $\sigma$ or $\tau$. The drift rate parameters show a similar but not identical pattern. The starting points ( $A$ and $z$ ) agree only with respect to $\mu$. Given that the LBA and the diffusion model are different accounts of cognitive processes, the level of alignment between the two is remarkable. Most importantly, the associations between the ex-Gaussian and LBA parameters were found to be non-unique. These results converge on the conclusion that one cannot unambiguously map the ex-Gaussian parameters to those of either of the evidence accumulation models.

\section{Study 2}

In this study I present a concrete empirical illustration of the simulations results by applying the LBA analyses to the same data sets used by Matzke and Wagenmakers (2009). These data are from two lexical decision experiments by Wagenmakers et al. (2008). In the first experiment ( $N=$ 17 ), task difficulty was manipulated by varying word fre- quency (high, low, and very low), and response caution was manipulated by emphasizing either response speed or response accuracy. The experiment consisted of a 3 (word frequency) x 2 (speed-accuracy level) design. Wagenmakers et al. (2008) fit the diffusion model and found the effect of word frequency to be accounted for by changes in drift rate $(v)$ and the effects of speed-accuracy instructions by changes in boundary separation (a). In a second experiment ( $N=19)$, task difficulty was manipulated again by varying word frequency, and in addition response bias was manipulated by varying the proportion of word to nonword stimuli in a list (i.e., $75 \%$ words or $75 \%$ nonwords). These resulted in a 3 (word frequency) x 2 (word-nonword proportion) design. In this experiment the effect of difficulty (i.e., frequency) was exclusively accounted for by changes in drift rate (v) and the effect of response bias (i.e., word non-word proportion) was associated with changes in starting point (z), such that the starting point $\mathrm{z}$ was higher in the $75 \%$ word condition than in the $75 \%$ nonword condition.

Fitting the ex-Gaussian to these data, Matzke and Wagenmakers (2009) reached the conclusion that: "the exGaussian parameters do not respond selectively to the effects of the word frequency, speed-accuracy, and proportion manipulations." (p.809). The $\mu$ was sensitive to all three experimental manipulations: speed-accuracy emphasis, word-nonword proportion, and word frequency. Since each manipulation is affecting a single diffusion model parameter, they concluded that $\mu$ was sensitive to changes in all three diffusion model parameters [drift rate (v), boundary separation (a), and starting point (z)]. The $\sigma$ was moderately influenced by word frequency and therefore by drift rate (v). The $\tau$ was sensitive to changes in both word frequency and speed accuracy emphasis and therefore by drift rate and boundary separation. (see p.809).

Using the same published data sets by Wagenmakers et al. (2008), I tested whether the experimental manipulations that had exerted selective influence on the diffusion model parameters exhibit similar selective influence on the LBA parameters. In addition, I tested whether and how the ex-Gaussian parameters are associated with the LBA parameters in these data. I first fit the ex-Gaussian parameters to the two experiments using the same methods reported in study 1 . After deriving the best fitting exGaussian parameters for each participant in each experimental condition, I conducted three separate ANOVAs on the means of the three ex-Gaussian parameters (i.e., $\mu, \sigma, \tau$ ) to assess the influence of the experimental manipulations on these parameters. The results of the ANOVA analyses are presented in Table 3. They replicate those of Matzke and Wagenmakers (2009), such that in the first Experiment (see Figure 5A), word frequency and speed accuracy in- 
Table 1 - Range of parameters used in the simulations. $v_{1}$ is the mean drift rate for the first accumulator, $v_{2}$ is the mean drift rate for the second accumulator. In the first version of the simulations (Figure 3), the mean drift rates sum up to one $\left(v_{1}+v_{2}=1\right)$. In the second version of the simulations (Figure 4), the mean drift $v_{2}$ was held fixed and the mean drift rates did not sum up to 1 (i.e., $v_{1}+v_{2} \neq 1$ ).

\begin{tabular}{lcccccc}
\hline Model & $b^{\smile} A$ & $A$ & $T_{e r}$ & $s$ & $v_{1}$ & $v_{2}$ \\
\hline Min & 0 & 0.15 & 0.1 & 0.15 & 0.5 & - \\
Max & 0.5 & 0.45 & 0.4 & 0.35 & 1 & - \\
Default & 0.12 & 0.25 & 0.25 & 0.27 & 0.74 & 0.2 \\
\hline
\end{tabular}

Table 2 - The association between parameters of the Ex-Gaussian distributions and parameters of the Linear Ballistic Accumulator (LBA) (left) and Diffusion model (right).

\begin{tabular}{|c|c|c|c|c|c|c|c|c|}
\hline \multirow[b]{2}{*}{$\begin{array}{l}\text { Ex-Gaussian } \\
\text { Parameter }\end{array}$} & \multicolumn{4}{|c|}{ LBA parameters } & \multicolumn{4}{|c|}{$\begin{array}{c}\text { Diffusion model parameters } \\
\text { (Matzke \& Wagenmakers, 2009) }\end{array}$} \\
\hline & $b$ & $A$ & $T_{e r}$ & $v$ & $a$ & $z$ & $T_{e r}$ & $v$ \\
\hline$\mu$ & ++ & - & ++ & - & ++ & - & ++ & - \\
\hline$\sigma$ & + & + & $\mathrm{x}$ & $\mathrm{x}$ & + & - & $\mathrm{x}$ & - \\
\hline$\tau$ & ++ & $\mathrm{x}$ & $\mathrm{x}$ & - & ++ & - & $\mathrm{x}$ & - \\
\hline
\end{tabular}

Note. ${ }^{++}$, substantial positive association; +, weak positive association; -, substantial negative association; -, weak negative association; $\mathrm{x}$, no association.

structions influenced all three parameters: $\mu, \sigma$, and $\tau$ increased as word frequency decreased. Similarly, higher $\mu$ and $\tau$ values were found in accuracy compared to speed instructions. In Experiment 2 (see Figure 5B) word frequency strongly influenced $\tau$, and to a lesser degree $\mu$. In addition, words proportion affected $\mu$ and $\sigma$, but not $\tau$.

Next, I fit the LBA model to the the RTs of each participant's data individually, excluding individual RTs according to the criteria of the original studies. The drift rate $(v)$, starting point $(A)$, boundary $(b)$ and non-decision time $\left(T_{e r}\right)$ were free to vary across the experiment conditions. In the data of Experiment 1 there were 12 conditions composed of 2 between-block levels of speed vs. accuracy emphasis instruction, and 6 within-block levels of word-frequency, which were themselves created by the factorial design of 3 levels of word frequency (high, low, very low), and 2 levels of stimuli-type (word, non-word). In the data of Experiment 2 there were also 12 conditions, which were composed of 2 between-block levels of word/non-word proportion (75\% word vs. $75 \%$ nonword), and 6 within-block levels of word-frequency, as in Experiment 1. In fitting both data sets the LBA models consisted of 49 free parameters. There were 4 LBA parameters ( $v, b$, $A, T_{e r}$ ) free to vary across 12 conditions and an additional parameter $(s)$ that was held fixed. The models were fit by minimizing the QMPE applied to the 0.1, 0.3, 0.5, 0.7, and 0.9 quantiles, using SIMPLEX algorithm.

The means of the best fitting parameters are presented in Figure 6A and 6B. Statistical inferences were supported by ANOVA (see Table 3). Note that conducting ANOVA on the diffusion and LBA parameters is a common practice (see Donkin, Brown, \& Heathcote, 2011; Wagenmakers et al., 2008).

The manipulation of speed vs. accuracy instructions emphasis affected only the boundary parameter (b), such that the speed condition was associated with a lower boundary than the accuracy condition. Donkin, Brown, and Heathcote (2011) have used the value of $b^{\triangleleft} A / 2$ as the most directly comparable quantity to the response threshold a of the diffusion model. Analysis on this quantity showed that it was indeed affected exclusively by the manipulation of instructions. Word frequency affected drift rate $(v)$ and starting point $(A)$ in both experiments. Note that if $b$ and $A$ are replaced by the (single) response caution parameter $b^{\triangleleft} A / 2$ selective influence holds for this manipulation. The word/nonword proportion affected starting point $(A)$ and non-decision time $\left(T_{e r}\right)$. Taking together, the results entail that in general, selective influence held for the LBA parameters, and therefore, the LBA can be used to test the unique correspondence of the ex-Gaussian parameters.

The association between the ex-Gaussian and LBA parameters can be inferred indirectly by identifying the experimental manipulations that affected both. A similar logic has been used by Matzke and Wagenmakers (2009) and by Rieger and Miller (2019). The results show that $\mu$ and $\sigma$ were affected by the three experimental manipulations, and are therefore likely associated with the three 
Figure 3 - Relations between the ex-Gaussian and LBA parameters revealed by simulations with the mean drift rates for two accumulators summing up to 1 (i.e., $v_{1}+v_{2}=1$ ).
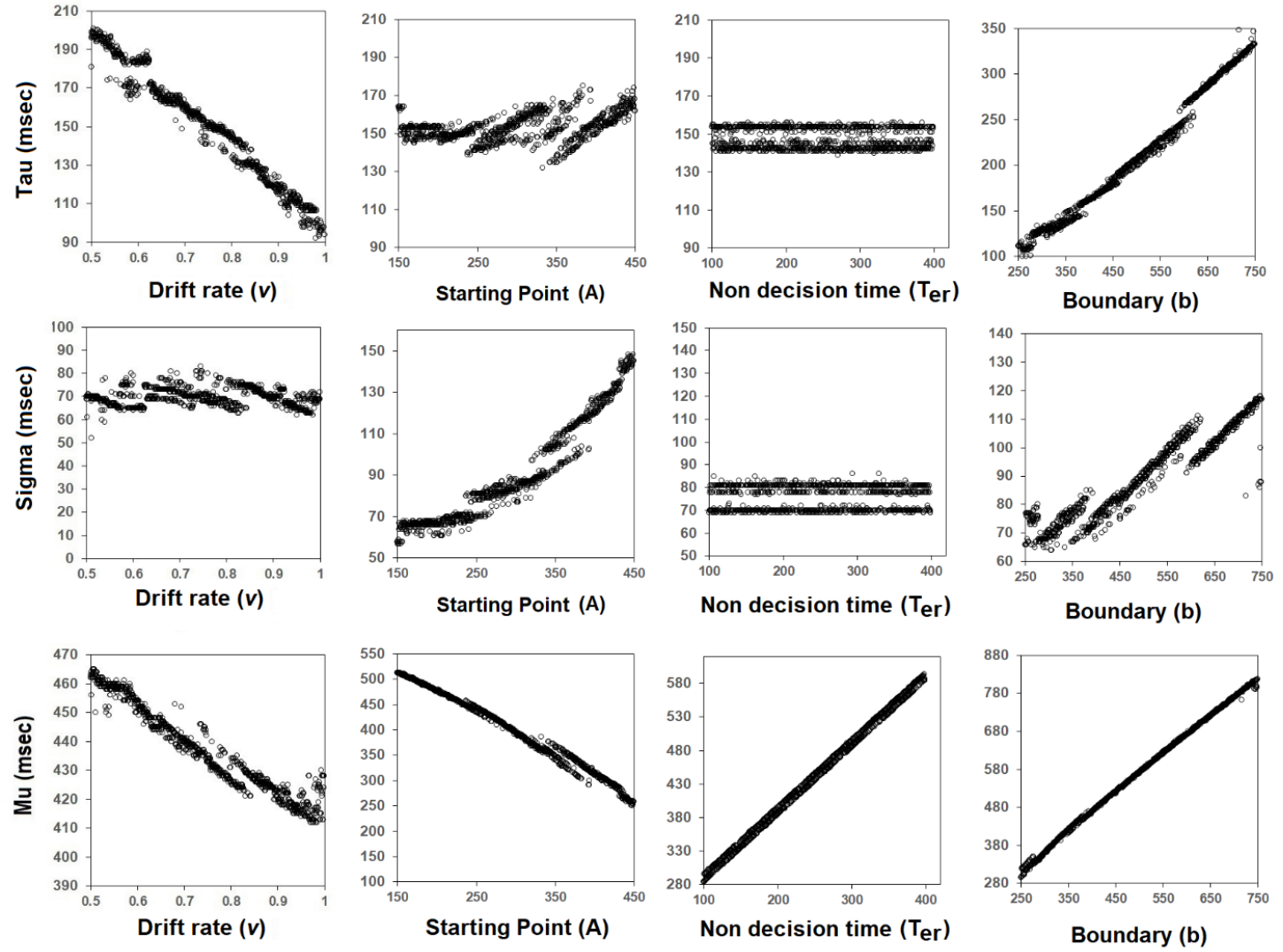

LBA quantities: $v, b-A / 2$, and $T_{e r}$. In addition, $\tau$ was affected by word frequency and speed-accuracy instructions, and is likely associated with $\mathrm{v}$ and $\mathrm{b}$. The empirical findings are therefore supported by the numerical simulations of Study 1.

Taken together, the results from the two studies converged on the conclusion that while the ex-Gaussian parameters maintain lawful relations with the parameters of the LBA, those relations are not unique. The two classes of parameters do not map in a one-to-one fashion. These results accord well with earlier findings by Matzke and Wagenmakers (2009) and by Rieger and Miller (2019).

\section{General Discussion}

The numerical simulations and the analysis of empirical data conducted in two studies lead us to five main conclusions: (a) the LBA parameters are associated with the ex-Gaussian parameters in a lawful way, (b) the correspondence is not unique because a single ex-Gaussian parameter maps to more than one LBA parameter and vice versa, (c) this correspondence is similar to that found with the diffusion model by Matzke and Wagenmakers (2009), (d) the experimental manipulations that exert selective influence on the diffusion model similarly affect the LBA parameters, and (e) the ex-Gaussian parameters do not map exclusively to the cognitive operations mimicked in the LBA parameters.

How does this study inform research on the exGaussian distribution? The current study shows that the ex-Gaussian parameters do not map uniquely to the LBA parameters, and it strengthen previous studies who reached similar conclusions with respect to the diffusion and EZ-diffusion parameters (Matzke \& Wagenmakers, 2009; Rieger \& Miller, 2019). One conclusion that can be drawn from these studies is that researchers should be cautious when using and interpreting the ex-Gaussian parameters in terms of cognitive stages or operations. Matzke and Wagenmakers (2009) and Rieger and Miller (2019) recommended their readers to completely withdraw from the deployment of the ex-Gaussian as a theoretical model, and 
Figure 4 - Relations between the ex-Gaussian and LBA parameters revealed by simulations with the mean drift rates for two accumulators not summing up to 1 (i.e., $v_{1}+v_{2} \neq 1$ ).
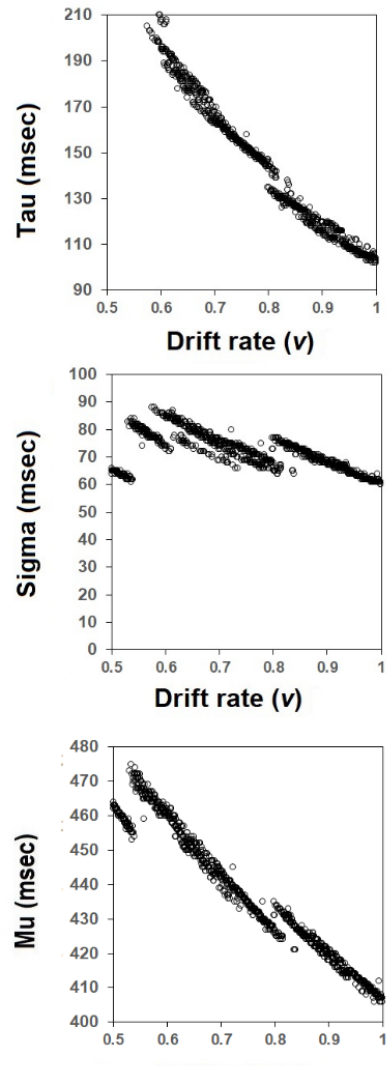

Drift rate $(v)$
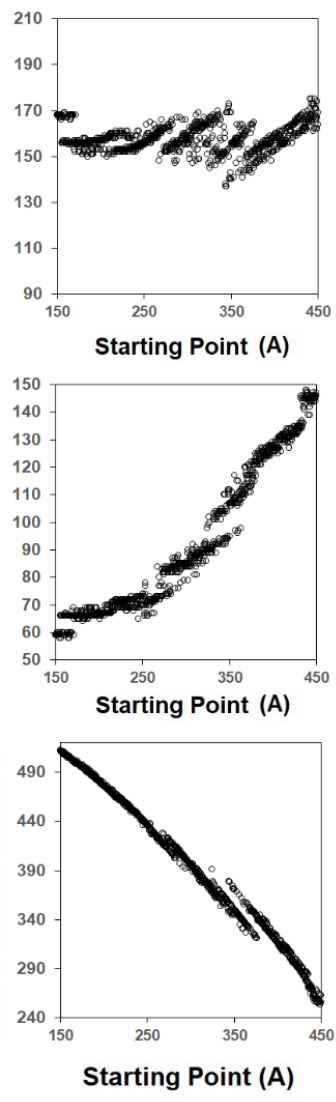
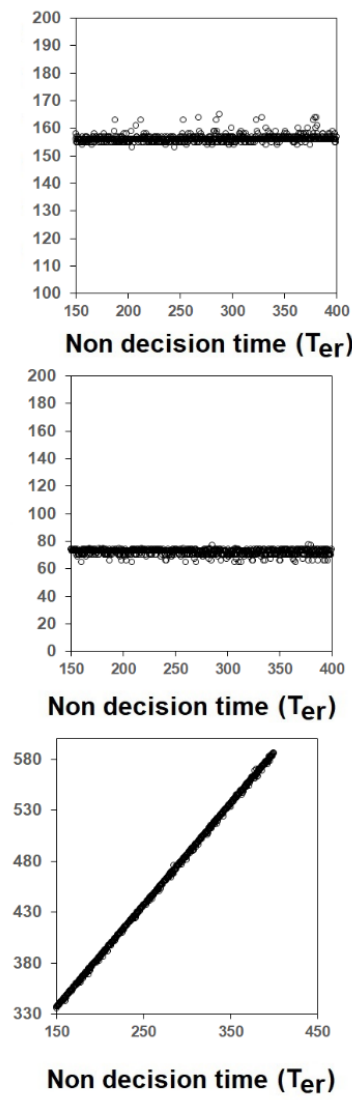

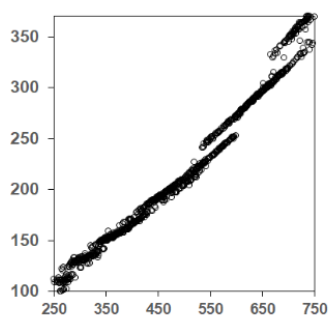

Boundary (b)
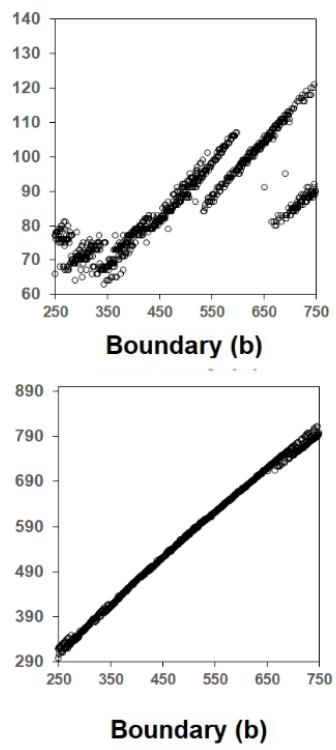

they encouraged researchers to keep deploying it as descriptive tool. While endorsing the latter recommendation, I do not share the former wholesale rejection of the exGaussian as a theoretical account due to the following reasons. First, the evidence coming from the sequential sampling models (the current study included) can speak only to these models. There might exist other cognitive models (currently unknown to us or not tested for this property) whose parameters do map uniquely to those of the ex-Gaussian. As a result, one cannot categorically reject the theoretical utility of the ex-Gaussian based on only one class of models. Second, the recommendation against using the ex-Gaussian parameters as a psychological account is coming mainly from the sequential sampling models and is based on a critical assumption that their parameters are selectively influenced by distinct experimental manipulations (Voss et al., 2004). However, if this assumption turns out to be invalid, then the recommendation is not valid anymore. A recent study by Rae, Heathcote, Donkin, Averell, and Brown (2014) demonstrated a failure of selective influence in both the diffusion and LBA models. In their study, the experimental manipulation of emphasizing speed versus accuracy, which is considered to selectively influence boundary parameters (Voss et al., 2004), has also been found to influence the drift rate parameters (see also Vandekerckhove, Tuerlinckx, \& Lee, 2011; Starns \& Ratcliff, 2010). If the assumption of selective influence is incorrect, then the results coming from the Matzke and Wagenmakers (2009), the Rieger and Miller (2019), and the current effort, should not be taken as strong evidence against the psychological validity of the ex-Gaussian parameters.

Third, it is well known that the estimation of parameters can introduce correlations between estimated values, a problem known as parameter dependency. This problem can afflict both estimation in the diffusion model (Ratcliff \& Tuerlinckx, 2002) and the ex-Gaussian model (Spieler,

\footnotetext{
${ }^{3}$ Ratcliff and Tuerlinckx (2002) have argued that: “a potentially major problem in recovering parameters for a model is that the value of the estimate
} 
Table 3 - ANOVA results on the best fitting ex-Gaussian parameters for experiment 1 and experiment 2 of Wagenmakers, Ratcliff, Gomez, and McKoon (2008). S = Speed Accuracy instructions (speed, accuracy), Fr = word frequency (High, Low, Very low), $\mathrm{W}=$ word/nonword proportion (75\% word, $75 \%$ nonword)

\begin{tabular}{|c|c|c|c|c|c|c|c|c|c|}
\hline & \multicolumn{3}{|c|}{$\mu$} & \multicolumn{3}{|c|}{$\sigma$} & \multicolumn{3}{|c|}{$\tau$} \\
\hline & MSE & $F$ & $p$ & MSE & $F$ & $p$ & MSE & $F$ & $p$ \\
\hline$S$ & 66579 & 2857 & $* * *$ & 2904 & 489 & $* * *$ & 408869 & 8232 & $* * *$ \\
\hline$F r$ & 16801 & 1090 & $* * *$ & 2501 & 329 & $* * *$ & 27368 & 1105 & $* * *$ \\
\hline$S \times F r$ & 2650 & 394 & $* * *$ & 22 & 5.19 & * & 2212 & 174 & $* * *$ \\
\hline$W$ & 218816 & 9327 & $* * *$ & 6120 & 141 & $* * *$ & 7607 & 96.95 & $* * *$ \\
\hline$F r$ & 9853 & 440 & $* * *$ & 1378 & 34.23 & $* * *$ & 134685 & 4597 & $* * *$ \\
\hline$W \times F r$ & 295 & 25 & $* * *$ & 2002 & 54.56 & $* * *$ & 2691 & 107 & $* * *$ \\
\hline
\end{tabular}

Note. ${ }^{*} \mathrm{p}<0.05,{ }^{* *} \mathrm{p}<0.01,{ }^{* * *} \mathrm{p}<0.005$

Table 4 - ANOVA results on the best fitting LBA parameters for experiment 1 and experiment 2 of Wagenmakers, Ratcliff, Gomez, and McKoon (2008). S = Speed Accuracy instructions (speed, accuracy), Fr = word frequency (High, Low, Very low), $\mathrm{W}=$ word/nonword proportion $(75 \%$ word, $75 \%$ nonword $)$

\begin{tabular}{|c|c|c|c|c|c|c|c|c|c|c|c|c|c|c|c|}
\hline & \multicolumn{3}{|c|}{$v$} & \multicolumn{3}{|c|}{$b$} & \multicolumn{3}{|c|}{$A$} & \multicolumn{3}{|c|}{$T_{e r}$} & \multicolumn{3}{|c|}{$b-A / 2$} \\
\hline & MSE & $F$ & $p$ & MSE & $F$ & $p$ & MSE & $F$ & $p$ & MSE & $F$ & $p$ & MSE & $F$ & $p$ \\
\hline$S$ & 0.10 & 3.4 & & 688146 & 29.9 & $* * *$ & 1167 & 0.07 & & 33518 & 3.9 & & 660100 & 30.2 & $* * *$ \\
\hline$F r$ & 1.07 & 36.5 & $* * *$ & 3833 & 0.23 & & 36380 & 4.20 & * & 8071 & 1.32 & & 24261 & 1.88 & \\
\hline$S \times F r$ & 0.12 & 4.3 & $*$ & 50112 & 3.68 & * & 11737 & 1.64 & & 1292 & 0.17 & & 32695 & 2.12 & \\
\hline$W$ & 0.16 & 2.0 & & 19737 & 0.31 & & 221497 & 9.6 & $* *$ & 345510 & 27.4 & $* * *$ & 1441229 & 3.5 & \\
\hline$F r$ & 1.97 & 31.2 & $* * *$ & 3578 & 0.13 & & 184392 & 14.5 & $* * *$ & 11538 & 1.88 & & 74530 & 2.6 & \\
\hline$W \times F r$ & 0.44 & 8.3 & $* *$ & 102144 & 3.30 & $*$ & 22062 & 1.5 & & 12751 & 1.44 & & 137473 & 4.0 & $*$ \\
\hline
\end{tabular}

Note. ${ }^{*} \mathrm{p}<0.05,{ }^{* *} \mathrm{p}<0.01,{ }^{* * *} \mathrm{p}<0.005$.

2001). ${ }^{3}$ It can lead to the creation of non-unique relations between the ex-Gaussian and the diffusion or LBA models. The upshot is that a categorical rejection of the exGaussian distribution as a psychological account is unjust at this stage. However, the current and previous studies can contribute to the understanding of these models, and the relations between their parameters.

Fourth, a theoretical approach that is based on converging operations (Garner, Hake, \& Eriksen, 1956; see also Algom \& Fitousi, 2016; Fitousi, 2015; Fitousi \& Wenger, 2013; Von Der Heide, Wenger, Bittner, \& Fitousi, 2018) can be used to remedy the problem. According to Garner et al. (1956), converging operations are: "any set of two or more experimental operations which allow the selection or elimination of alternative hypotheses or concepts which could explain an experimental result. They are called converging operations because they are not perfectly correlated and thus can converge on a single concept" (Garner et al., 1956, p. 150-151). An excellent example to the converging operation method appears in the study by Schmiedek et al. (2007), in which they relate the parameters of the exGaussian distribution and those of the diffusion model to constructs of working memory. These authors have tested simultaneously several constraining hypotheses with respect to the theoretical concept of working memory. In their investigation models of latent variables revealed important relations of the ex-Gaussian parameters with other high cognitive functions. The upshot is that statistical regularities in the ex-Gaussian parameters might be correlated with theoretical constructs, allowing a psychological interpretation (see Fitousi, 2020a).

How does this study inform research on the LBA and diffusion models? The current study sheds light on the question of whether the LBA and the diffusion models produce converging or diverging inferences. The evidence so far has been mixed. van Ravenzwaaij and Oberauer (2009) did not find simple one-to-one mapping between the LBA and diffusion model parameters. Osth et al. (2017) have presented a concrete research question in which the LBA and diffusion models lead to different conclusions. Donkin, Brown, Heathcote, and Wagenmakers (2011) have cross fitted data from the two models and found that: "when data were generated from the diffusion model, changes in response caution affected drift rate and non-

for one parameter may be significantly correlated with the value of another” (p. 452). To investigate how serious this problem, these authors computed correlations between each pair of the 6 diffusion-model parameters based on 100 fits to simulated data. They did find many cases of positive correlations between variables (see their Figure 6). They concluded that: "This means that if the sizes of differences among parameters values are important, correlations must be considered” (p.455). 
Figure 5 - Best fitting values of the ex-Gaussian parameters: $\mu, \sigma, \tau$ for the data sets of the Wagenmakers, Ratcliff, Gomez, and McKoon (2008) in Experiment 1 (Panel A) and Experiment 2 (Panel B). HF, high-frequency words; LF, low-frequency words, VLF, very-low-frequency words.
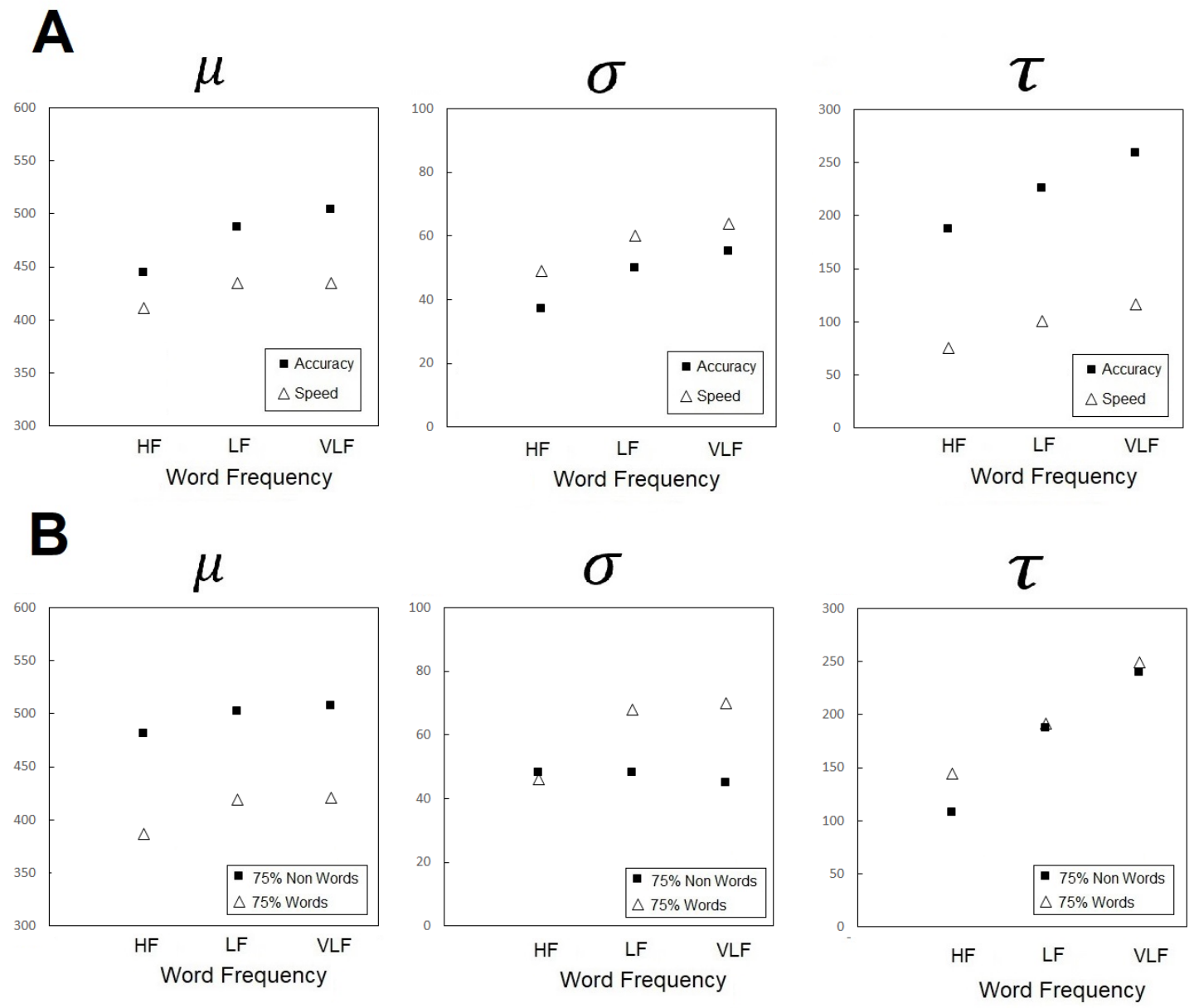

decision time in the LBA, while response caution changes in data generated from the LBA model caused changes only in non-decision time for the diffusion model.” (p.65). Donkin, Brown, and Heathcote (2011) also argued for differences in the shape of the RT distributions generated by the two models. However, a recent study by Dutilh et al. (2019) used a collaborative, blinded, validation study in which seventeen research groups analyzed fourteen data sets using different evidence accumulation models. The results showed that different groups reached a strong consensus regarding the experimental manipulation that generated the data. However, those who were using the diffusion model (except for the EZ) suggested an effect of changes in non-decision time much more than those who were using the LBA, and there were also discrepancies in the detection of response bias. Dutilh et al concluded that although modelers reach similar conclusion their 'degrees of freedom' did affect their conclusions. It might be the case that the parameters of the LBA and diffusion model do not map in a one-to-one correspondence, but the current study shows that when they are tested against a third model (i.e. ex-Gaussian), they exhibit a high degree of functional similarity, such that parameters evinced comparable sensitivity to changes in the ex-Gaussian parameters and related experimental manipulations. But these relations are not necessarily unique.

\section{References}

Algom, D., Eidels, A., Hawkins, R. X. D., Jefferson, B., \& Townsend, J. T. (2015). Features of response times: 
Figure 6 - Means of best fitting LBA parameters of the Wagenmakers, Ratcliff, Gomez, and McKoon (2008) Experiment 1 (top panel) and Experiment 2 (bottom panel). Error bars are standard error of the mean.

A

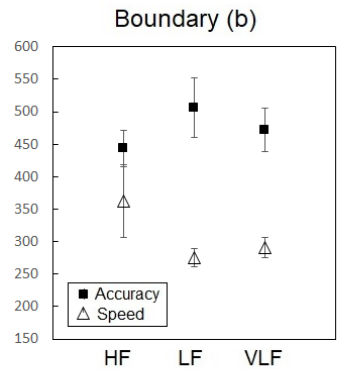

Word Frequency

B

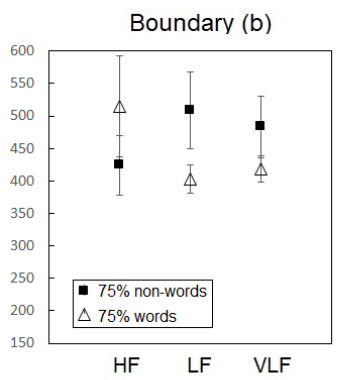

Word Frequency

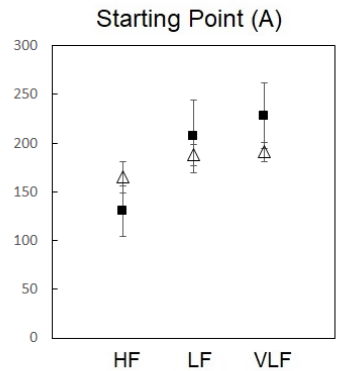

Word Frequency

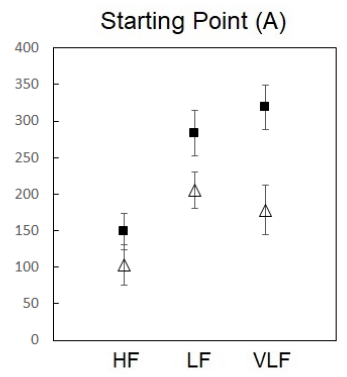

Word Frequency

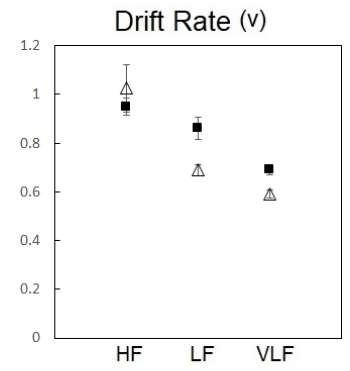

Word Frequency

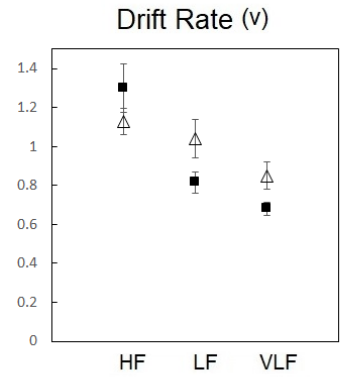

Word Frequency

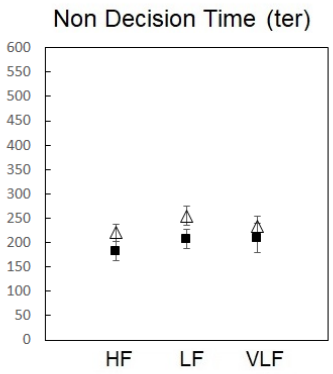

Word Frequency

Non Decision Time (ter)

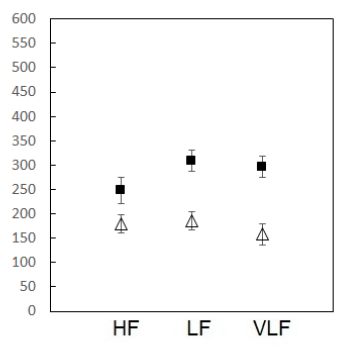

Word Frequency

Identification of cognitive mechanisms through mathematical modeling. In J. R. Busemeyer, J. T. Townsend, Z. Wang, \& A. Eidels (Eds.), Oxford handbook of computational and mathematical psychology (pp. 63-98). New York, NY: Oxford University Press.

Algom, D., \& Fitousi, D. (2016). Half a century of research on garner interference and the separabilityintegrality distinction. Psychological Bulletin, 142, 1352-1383. doi:10.1037/bul0000072

Balota, D. A., \& Spieler, D. H. (1999). Word frequency, repetition, and lexicality effects in word recognition tasks: Beyond measures of central tendency. Journal of Experimental Psychology: General, 128, 32-55. doi:10 . 1037/0096-3445.128.1.32

Balota, D. A., \& Yap, M. J. (2011). Moving beyond the mean in studies of mental chronometry: The power of response time distributional analyses. Current Directions in Psychological Science, 20, 160-166. doi:10 . 1177/0963721411408885

Balota, D. A., Yap, M. J., Cortese, M. J., \& Watson, J. M. (2008). Beyond mean response latency: Response time distributional analyses of semantic priming. Journal of

Memory and Language, 59(4), 495-523. doi:10.1016/j. jml.2007.10.004

Boehm, U., Marsman, M., Matzke, D., \& Wagenmakers, E.-J. (2018). On the importance of avoiding shortcuts in applying cognitive models to hierarchical data. Behavior Research Methods, 50, 1614-1631. doi:10.3758/s13428018-1054-3

Brown, S. D., \& Heathcote, A. (2008). The simplest complete model of choice response time: Linear ballistic accumulation. Cognitive Psychology, 57, 153-178. doi:10 . 1016/j.jml.2007.10.004

Buzy, W. M., Medoff, D. R., \& Schweitzer, J. B. (2009). Intraindividual variability among children with ADHD on a working memory task: An ex-Gaussian approach. Child Neuropsychology, 15(5), 441-459. doi:10.1080/ 09297-04080-2646991

Coyle, T. R. (2003). A review of the worst performance rule: Evidence, theory, and alternative hypotheses. Intelligence, 31, 567-587. doi:10.1016/S0160-2896(03)000540

Donkin, C., Brown, S., \& Heathcote, A. (2011). Drawing conclusions from choice response time models: A tuto- 
rial using the linear ballistic accumulator. Journal of Mathematical Psychology, 55(2), 140-151. doi:10.1016/ j.jmp.2010.10.001

Donkin, C., Brown, S., Heathcote, A., \& Wagenmakers, E.-J. (2011). Diffusion versus linear ballistic accumulation: Different models but the same conclusions about psychological processes? Psychonomic Bulletin \& Review, 18(61-69), 00. doi:10.3758/s13423-010-

Dutilh, G., Annis, J., Brown, S. D., Cassey, P., Evans, N. J., Grasman, R. P., ... Kupitz, C. N. (2019). The quality of response time data inference: A blinded, collaborative assessment of the validity of cognitive models. Psychonomic Bulletin \& Review, 26, 1051-1061. doi:10. 3758/s13423-017-1417-2

Evans, N. J. (2019). Assessing the practical differences between model selection methods in inferences about choice response tasks. Psychological Bulletin \& Review, 26, 1070-1098. doi:10.3758/s13423-018-01563-9

Evans, N. J., Holmes, W. R., \& Trueblood, J. S. (2019). Response-time data provide critical constraints on dynamic models of multi-alternative, multi-attribute choice. Psychonomic Bulletin \& Review, 26(3), 901-933.

Evans, N. J., \& Servant, M. (2020). A comparison of conflict diffusion models in the flanker task through pseudolikelihood Bayes factors. Psychological Review, 127(1), 114-135.

Fitousi, D. (2015). Composite faces are not processed holistically: Evidence from the garner and redundant target paradigms. Attention, Perception, \& Psychophysics, 77(6), 2037-2060. doi:10.3758/s13414-015-0887-4

Fitousi, D. (2020a). Decomposing the composite face effect: Evidence for non-holistic processing based on the ex-Gaussian distribution. Quarterly Journal of Experimental Psychology, in press, 1-20.

Fitousi, D., \& Wenger, M. J. (2013). Variants of independence in the perception of facial identity and expression. Journal of Experimental Psychology: Human Perception, and Performance, 39, 133-155. doi:10.1037/ a0028001

Forstmann, B. U., Ratcliff, R., \& Wagenmakers, E.-J. (2016). Sequential sampling models in cognitive neuroscience: Advantages, applications, and extensions. Annual Review of Psychology, 67, 641-666. doi:10 . 1146/annurev-psych-122414-03364

Garner, W. R., Hake, H. W., \& Eriksen, C. W. (1956). Operationism and the concept of perception. Psychological Review, 63(3), 149-159.

Gordon, B., \& Carson, K. (1990). The basis for choice reaction time slowing in alzheimer's disease. Brain \& Cognition, 13, 148-166. doi:10.1016/0278-2626(90)90047-R

Heathcote, A., Popiel, S. J., \& Mewhort, D. J. (1991). Analysis of response time distributions: An example us- ing the stroop task. Psychological Bulletin, 109(2), 340347. doi:10.1037/0033-2909.109.2.340

Hockley, W. E. (1984). Analysis of response time distributions in the study of cognitive processes. Journal of Experimental Psychology: Learning, Memory, and Cognition, 10, 598-615. doi:10.1037/0278-7393.10.4.598

Hohle, R. H. (1965). Inferred components of reaction times as functions of foreperiod duration. Journal of Experimental Psychology, 69(4), 740. doi:10.1037/h0021

Jackson, J. D., Balota, D. A., Duchek, J. M., \& Head, D. (2012). White matter integrity and reaction time intraindividual variability in healthy aging and early-stage alzheimer disease. Neuropsychologia, 50(3), 357-366. doi:10.1016/j.neuropsychologia.2011.11.024

Kóbor, A., Takács, Á., Bryce, D., Szucs, D., Honbolygó, F., ... Csépe, V. (2015). Children with ADHD show impairments in multiple stages of information processing in a stroop task: An ERP study. Developmental Neuropsychology, 40(2015), 329-347. doi:10 . 1080 / 87565641 . 2015.1086770

Leth-Steensen, C., Elbaz, Z. K., \& Douglas, V. I. (2000). Mean response times, variability, and skew in the responding of ADHD children: A response time distributional approach. Acta Psychologica, 104(2), 167-190. doi:10. 1016/S0001-6918(00)00019-6

Logan, G. D. (1988). Toward an instance theory of automatization. Psychological Review, 95(4), 492-527. doi:10. 1037/0033-295X.95.4.492

Luce, R. D. (1986). Response times: Their role in inferring elementary mental organization. Oxford: Oxford University Press.

Matzke, D., \& Wagenmakers, E.-J. (2009). Psychological interpretation of the ex-Gaussian and shifted wald parameters: A diffusion model analysis. Psychonomic Bulletin \& Review, 16(5), 798-817. doi:10.3758/PBR. 16.5.798

Nelder, J. A., \& Mead, R. (1965). A simplex method for function minimization. The Computer Journal, 7(4), 308313. doi:10.1093/comjnl/7.4.308

Osth, A. F., Bora, B., Dennis, S., \& Heathcote, A. (2017). Diffusion vs. linear ballistic accumulation: Different models, different conclusions about the slope of the zroc in recognition memory. Journal of Memory and Language, 96, 36-61. doi:10.1016/j.jml.2017.04.003

Possamaï, C.-A. (1991). A responding hand effect in a simple-rt precueing experiment: Evidence for a late locus of facilitation. Acta Psychologica, 77, 47-63. doi:10.1016/0001-6918(91)90064-7

Rae, B., Heathcote, A., Donkin, C., Averell, L., \& Brown, S. (2014). The hare and the tortoise: Emphasizing speed can change the evidence used to make decisions. Journal of Experimental Psychology: Learning, 
Memory, and Cognition, 40(5), 1226-1243. doi:10.1037/ a0036801

Ratcliff, R. (1978). A theory of memory retrieval. Psychological Review, 85(2), 59-108. doi:10.1037/0033-295X.85. 2.59

Ratcliff, R., \& Murdock, B. B. (1976). Retrieval processes in recognition memory. Psychological Review, 83, 190214. doi:10.1037/0033-295X.83.3.190

Ratcliff, R., \& Smith, P. L. (2004). A comparison of sequential sampling models for two-choice reaction time. Psychological review, 111(2), 333-367. doi:10.1037/0033295X.111.2.333

Ratcliff, R., Smith, P. L., Brown, S. D., \& McKoon, G. (2016). Diffusion decision model: Current issues and history. Trends in Cognitive Sciences, 20(4), 260-281. doi:10 . 1016/j.tics.2016.01.007

Ratcliff, R., \& Tuerlinckx, F. (2002). Estimating parameters of the diffusion model: Approaches to dealing with contaminant reaction times and parameter variability. Psychonomic Bulletin \& Review, 9, 438-481. doi:10. 3758/BF03196302

Rieger, T., \& Miller, J. (2019). Are model parameters linked to processing stages? an empirical investigation for the ex-Gaussian, ex-Wald, and EZ diffusion models. Psychological Research, online, 1-17. doi:10 . 1007 / s00426-019-01176-4

Rohrer, D. (1996). On the relative and absolute strength of a memory trace. Memory \& Cognition, 24(2), 00880. doi:10.3758/BF032

Rohrer, D., \& Wixted, J. T. (1994). An analysis of latency and interresponse time in free recall. Memory \& Cognition, 22(5), 98390. doi:10.3758/BF031

Rotello, C. M., \& Zeng, M. (2008). Analysis of rt distributions in the remember-know paradigm. Psychonomic Bulletin \& Review, 15, 825-832. doi:10.3758/PBR.15.4.825

Rouder, J. N., Lu, J., Speckman, P., Sun, D., \& Jiang, Y. (2005). A hierarchical model for estimating response time distributions. Psychonomic Bulletin \& Review, 12(2), 195-223. doi:10.3758/BF03257252

Schmiedek, F., Oberauer, K., Wilhelm, O., Süß, H.-M., \& Wittmann, W. W. (2007). Individual differences in components of reaction time distributions and their relations to working memory and intelligence. Journal of Experimental Psychology: General, 136(3), 414429. doi:10.1037/0096-3445.136.3.414

Schwarz, W. (2001). The ex-wald distribution as a descriptive model of response times. Behavior Research Methods, Instruments, \& Computers, 33(4), 457-469. doi:10.1037/e537102012-270

Spieler, D. H. (2001). Modeling age-related changes in information processing. European Journal of Cognitive Psychology, 13, 217-234. doi:10.1080/09541440125907
Starns, J. J., \& Ratcliff, R. (2010). The effects of aging on the speed-accuracy compromise: Boundary optimality in the diffusion model. Psychology and Aging, 25, 377390. doi:10.1037/a0018022

Steinhauser, M., \& Hübner, R. (2009). Distinguishing response conflict and task conflict in the stroop task: Evidence from ex-Gaussian distribution analysis. Journal of Experimental Psychology: Human Perception and Performance, 35, 1398-1412. doi:10 . 1037 / a0016467

Townsend, J. T., \& Ashby, F. G. (1983). Stochastic modeling of elementary psychological processes. Cambridge, MA.: Cambridge University Press.

Tse, C. S., Balota, D. A., Yap, M. J., Duchek, J. M., \& McCabe, D. P. (2010). Effects of healthy aging and early stage dementia of the alzheimer's type on components of response time distributions in three attention tasks. Neuropsychology, 24, 300-315. doi:10.1037/a0018274

Usher, M., \& McClelland, J. L. (2001). The time course of perceptual choice: The leaky, competing accumulator model. Psychological Review, 108, 550-592. doi:10. 1037/0033-295X.111.3.757

Van der Linden, W. J. (2006). A lognormal model for response times on test items. Journal of Educational and Behavioral Statistics, 31(2), 181-204.

van Ravenzwaaij, D., \& Oberauer, K. (2009). How to use the diffusion model: Parameter recovery of three methods: EZ, fast-dm, and DMAT. Journal of Mathematical Psychology, 53, 463-473. doi:10.1016/j.jmp.2009.09. 004

Vandekerckhove, J., Tuerlinckx, F., \& Lee, M. D. (2011). Hierarchical diffusion models for two- choice response times. Psychological Methods, 16(1), 44-62. doi:10 . 1037/a0021765

Von Der Heide, J., Wenger, M. R., Bittner, J., \& Fitousi, D. (2018). Converging operations and the role of perceptual and decisional influences on the perception of faces: Neural and behavioral evidence. Brain \& Cognition, 122, 59-75. doi:10.1016/j.bandc.2018.01.007

Voss, A., Rothermund, K., \& Voss, J. (2004). Interpreting the parameters of the diffusion model: An empirical validation. Memory \& Cognition, 32, 1206-1220. doi:10. 3758/BF03196893

Wagenmakers, E.-J., Ratcliff, R., Gomez, P., \& McKoon, G. (2008). A diffusion model account of criterion shifts in the lexical decision task. Journal of Memory and Language, 58, 140-159. doi:10.1016/j.jml.2007.04.00

Wagenmakers, E.-J., Van Der Maas, H. L., \& Grasman, R. P. (2007). An EZ-diffusion model for response time and accuracy. Psychonomic Bulletin \& Review, 14, 3-22. doi:10.3758/bf03194023 
Table 5 - Parameter recovery for the code to fit the parameters

\begin{tabular}{lcccc}
\hline & $\mu$ & $\sigma$ & $\tau$ & chi square \\
\hline Real & 200 & 50 & 100 & \\
Recovered & 201 & 56 & 98 & 37.25 \\
Real & 600 & 120 & 200 & \\
Recovered & 604 & 124 & 197 & 19.0 \\
Real & 200 & 80 & 500 & \\
Recovered & 190 & 80 & 496 & 18.3 \\
Real & 800 & 90 & 120 & \\
Recovered & 800 & 91 & 123 & 9.7 \\
Real & 550 & 30 & 50 & \\
Recovered & 551 & 31 & 49 & 21.6 \\
Real & 50 & 10 & 200 & \\
Recovered & 49.5 & 10.05 & 198.62 & 31.6 \\
\hline
\end{tabular}

\section{Appendix: Supplementary materials}

To fit the ex-Gaussian parameters to the RT distributions I wrote a MATLAB code. This code make use of the fminsearchbnd function in MATLAB, which is an optimization routine based on the SIMPLEX algorithm (Nelder \& Mead, 1965). To validate the algorithm, I run a parameter recovery simulation (attached to the supplementary files), in which I simulated RT distributions with known ex-Gaussian parameters, and then recovered those parameters from the data. Each row of Table 5 represents the first (and single) attempt at recovering the parameter values. As can be seen, the algorithm worked very well in recovering the real parameters from the data.

\section{Citation}

Fitousi, D. (2020b). Linking the Ex-Gaussian parameters to cognitive stages: Insights from the Linear Ballistic Accumulator (LBA) model. The Quantitative Methods for Psychology, 16(2), 91-106. doi:10.20982/tqmp.16.2.p091

Copyright ( 1 2020, Fitousi. This is an open-access article distributed under the terms of the Creative Commons Attribution License (CC BY). The use, distribution or reproduction in other forums is permitted, provided the original author(s) or licensor are credited and that the original publication in this journal is cited, in accordance with accepted academic practice. No use, distribution or reproduction is permitted which does not comply with these terms.

Received: 29/07/2019 Accepted: 03/03/2020 